\title{
Conception of a new binder containing plaster and sulfoaluminate clinker
}

\section{Etude d'un liant à base de plâtre et de clinker sulfoalumineux}

\author{
Moulay Larbi Abidi ${ }^{1}$; Nouzha Lamdouar ${ }^{1}$; Latifa Iraqi ${ }^{1}$; Hanane Moulay Abdelaali ${ }^{1}$; \\ Amina Alaoui ${ }^{2}$
}

${ }^{1}$ EMI : Eole Mohammadia d'ingénieurs Université Mohammed V Agdal, Rabat, Maroc.

${ }^{2}$ Ecole centrale des ponts et chaussées, Paris, France.

\begin{abstract}
This study aimed to produce an economic and ecological building material respecting the quality and safety common standards. Large wealth of gypsum even naturally in the careers or through phosphate industry has got in Morocco. After dehydration of gypsum, we obtain the plaster. This material is broadly used in the construction industry. The plaster is characterized by its good fire resistance, soundproofing and insulation and its decorative virtues. However, the principal limit of this substance is its water -keeping behavior. In order to resolve this problem, this study is focused on the conception of a new binder containing plaster and sulfoaluminate clinker. The treatement of the subject is based on the execution of three experimental plans. We will be lead into analyzing the relevance of the preparation of gypsum or plaster mortar and appreciate the impact of the sulfoaluminate clinker on the plaster.The optimization of the formulations of concreate containing plaster (or gypsum) and sulfoaluminate clinker would be introducing a several challenges which may be at the origin in a new era in the fields of civil engineering.
\end{abstract}

\begin{abstract}
Résumé. Ce travail a été réalisé dans le but de la production d'un matériau de construction à la fois économique et écologique respectant les normes en vigueur. Dans cette perspective, l'étude de la faisabilité d'un nouveau liant à base de plâtre et de clinker sulfoalumineux s'avère intéressante. Le plâtre est un matériau largement utilisé dans la construction, il se caractérise par sa bonne résistance au feu, sa bonne isolation acoustique et thermique et ses vertus décoratives. Or la limite principale de ce matériau se trouve dans se tenue à l'eau. Pour remédier à ce problème, l'idée est l'utilisation du clinker sulfoalumineux et l'étude de son impact sur un mortier de plâtre. Pour ce faire, le traitement du sujet s'est appuyé sur la réalisation de trois plans d'expérience en étudiant l'influence des facteurs eau/liant, teneur en CSA (clinker sulfoalumineux) du liant et nature du gypse. Ceci pour analyser la pertinence de la préparation de mortier de plâtre (ou de gypse) et d'apprécier l'impact du clinker sulfoalumineux au mortier de plâtre. Enfin, un logiciel de traitement de donnée statistique a été utilisé pour optimiser la formulation du liant à base de plâtre, cette optimisation doit prendre en considération les résistances mécaniques et les résistances à la tenue à l'eau.
\end{abstract}




\section{Introduction}

L'utilisation du gypse trouve sa place dans le domaine de la construction, la déshydratation de ce matériau, produit le plâtre, qui lui aussi, est très utilisé dans la construction, vu ces avantages multiples. Néanmoins, le plâtre présente un inconvénient par rapport à sa tenue à l'eau. Pour stabiliser ce matériau vis-à-vis à l'eau, l'étude d'un liant à base de plâtre et de clinker a été envisagée. Afin d'évaluer l'influence du clinker sur le plâtre et de mette à profit son utilisation future.

Des études réalisées sur l'élaboration d'un béton à base de gypse et de clinker Portland, ont montré que la réaction chimique entre le clinker Portland et le gypse produit une ettringite gonflante.

L'étude s'intéresse à l'élaboration d'un liant à base de plâtre et de clinker sulfoalumineux, dont l'hydratation en présence de gypse produit une ettringite primaire a priori stable.

L'objectif de cette étude est d'apprécier l'impact de l'ajout du clinker sulfoalumineux au mortier de plâtre sur sa résistance et sa tenue à l'eau, puis d'évaluer la nécessité de transformer le gypse en plâtre en adoptant les mêmes formulations dans la fabrication de mortiers de gypse et de plâtre.

\section{Définitions}

\subsection{Le gypse}

Le gypse ou sulfate de calcium est le plus commun des sulfates naturels. Il est à la base de la fabrication du plâtre. On distingue deux variétés de gypse : le gypse naturel et artificiel.

Le gypse naturel est une roche saline sédimentaire, il se trouve en quantité importante dans les dépôts d'évaporation avec le sel, l'anhydride et la marne.

Il est caractérisé par sa faible solubilité dans l'eau et Il a une densité moyenne de 2,32.

Le gypse chimique a deux origines : il provient de la fabrication de l'acide phosphorique utilisé dans la préparation d'engrais riche ou du traitement d'effluent contenant $\mathrm{H} 2 \mathrm{SO} 4$.

Pour transformer le gypse naturel ou chimique en plâtre, il faut procéder à un ensemble de phases : l'extraction, le concassage, le séchage, le deuxième concassage, la cuisson, le broyage, le blutage et enfin l'ensachage.

\subsection{Mortiers et béton de plâtre}

\subsubsection{Mortier de plâtre}

Le gonflement du mortier de plâtre est supérieur à celui des plâtres purs. Les résistances à la traction et à la compression sont supérieures à celles de plâtre pur, mais au delà de certaines limites de granulométrie et de proportions du mélange, les résistances diminuent à nouveau.

\subsubsection{Béton de plâtre}

Deux études ont été réalisées par Rémillon (1961-62) concernant un béton en plâtre aux graviers de grés et un autre aux graviers de gypse. Les résultats de ces études montrent que les agrégats de gypse présentent une meilleure résistance à la compression sèche (10Mpla), une meilleure résistance à la traction par flexion $(1,3$ Mpa) et un module d'élasticité linéaire de 15 Gpa.

\subsection{Clinker sulfoalumineux}

Le Clinker sulfoalumineux est un clinker spécial, obtenu à partir d'un mélange de bauxite, de calcaire et de sulfate de calcium cuit à $1250-1300^{\circ} \mathrm{C}$. Les principales composantes de ce matériau sont : la Yeelimite, la belite, la Ferrite et l'Aluminate de calcium.

Les avantages du ciment sulfoalumineux par rapport au ciment portland sont :

- Environnementale : car les réactions de clinkérisation $\mathrm{du}$ Clinker sulfoalumineux se produisent à une température inferieur à celles du clinker portland ce qui nécessite moins de consommation d'énergie fossile et par conséquent moins d'émission de gaz à effet de serre. De même pour l'énergie nécessaire au broyage du Clinker sulfoalumineux, elle est inferieur à celle nécessaire au broyage du Clinker portland.

- Mécanique : pour des rapports eau/ciment égaux et de degrés d'hydratation égaux, la porosité de la pâte préparée à partir de ciments sulfoalumineux est inférieure à celle du ciment portland. Aussi la réaction entre le clinker portland et le plâtre produit une ettringite gonflante par contre avec le clinker sulfoalumineux, il produit une ettringite qui peut être stable.

\section{Partie expérimentale}

\subsection{Procédure adoptée}

Dans le but d'établir une formulation d'un mortier à base de plâtre et l'étude de ses propriétés mécaniques et rhéologiques. L'étude s'est basée sur un mortier normalisé composé, d'une partie de ciment, pour trois parties de sable et le rapport eau/ciment variable.

La méthodologie adoptée est celle des plans d'expérience, c'est une méthode rigoureuse qui permet d'étudier la dépendance d'une mesure de facteurs qualitatifs et quantitatifs en optimisant le nombre d'expériences à réaliser.

Le mortier utilisé est constitué des matériaux suivants : Un Sable qui a un coefficient d'absorption 0.5 ; Un Gypse naturel «le Sulykal DH Bleu»; Un Clinker sulfoalumineux CSA; Un plâtre de $\mathrm{PH}=8.5$ et une eau potable de PH neutre.

Les propriétés mécaniques et rhéologique étudiées sont : la mesure du $\mathrm{PH}$; le Temps de prise; l'étalement et 
l'affaissement au mini cône, les essais mécaniques concernant les résistances en flexion et en compression à 2.7 et à 28 jours et les essais pour évaluer la tenue à l'eau concernant l'absorption capillaire et le vieillissement accéléré.

Les mélanges sont constitués comme suit :

- La quantité du sable est fixée à $1350 \mathrm{~g}$

- La masse du liant hydraulique est de $450 \mathrm{~g}$

- Le rapport E/L est égal à 0.6 ou 0.7

- Le pourcentage du clinker sulfoalumineux :0\% et $30 \%$ pour voir son influence sur le mortier de plâtre.

- La nature du gypse : plâtre gypse déshydraté ou gypse.

- Le rapport $\mathrm{S} /(\mathrm{S}+\mathrm{L})($ Sable/Sable+liant) : $0 \%$ et $75 \%$ pour comparer la pate de plâtre pur avec le mortier.

Le problème est traité en considérant 3 plans d'expériences à deux facteurs. 8 formules sont réalisées 6 avec le plâtre et 2 avec le gypse. Les formulations étudiées sont récapitulées dans le tableau ci après.

Table 1. Formulations étudiées.

\begin{tabular}{|c|c|c|c|c|c|c|c|}
\hline & $\begin{array}{l}\mathrm{E} / \\
\mathrm{L}\end{array}$ & $\begin{array}{l}\mathrm{CSA} / \\
\mathrm{L}\end{array}$ & $\begin{array}{l}\mathrm{S} / \\
(\mathrm{S}+\mathrm{L})\end{array}$ & $\begin{array}{l}\text { CSA } \\
(\mathrm{g})\end{array}$ & $\begin{array}{l}\text { Plâtre } \\
\text { (g) }\end{array}$ & $\begin{array}{l}\text { Eau } \\
(\mathrm{g})\end{array}$ & Sable $(\mathrm{g})$ \\
\hline \multicolumn{8}{|c|}{ Plan d'expérience $\mathrm{N}^{\circ} 1$} \\
\hline Formule 1 & 0.6 & 0 & $75 \%$ & 0 & 450 & 270 & 1350 \\
\hline Formule 2 & 0.7 & 0 & $75 \%$ & 0 & 450 & 315 & 1350 \\
\hline Formule 3 & 0.6 & $30 \%$ & $75 \%$ & 135 & 315 & 270 & 1350 \\
\hline Formule 4 & 0.7 & $30 \%$ & $75 \%$ & 135 & 315 & 315 & 1350 \\
\hline \multicolumn{8}{|c|}{ Plan d'expérience $\mathrm{N}^{\circ} 2$} \\
\hline Formule 5 & 0.6 & $30 \%$ & $75 \%$ & 135 & 315 & 270 & 1350 \\
\hline Formule 6 & 0.7 & $30 \%$ & $75 \%$ & 135 & 315 & 315 & 1350 \\
\hline \multicolumn{8}{|c|}{ Plan d'expérience $\mathrm{N}^{\circ} 3$} \\
\hline Formule 7 & 0.6 & - & 0 & 0 & 1800 & 1080 & 0 \\
\hline Formule 8 & 0.7 & - & 0 & 0 & 1800 & 1260 & 0 \\
\hline
\end{tabular}

\subsection{Essais réalisés et résultats}

La préparation du mortier est effectuée comme suit: Le sable à sec est mélangé manuellement, puis le clinker sulfoalumineux est ajouté, ensuite le plâtre est ajouté. Le tout est remué jusqu'à homogénéisation du mélange. Après préparation du mortier les essais sont réalisés sur mortier frais et sur mortier durcis.

Concernant le mortier frais les essais réalisés sont la mesure du pH des gâchées et les essais de rhéologie.

Les résultats de cet essais montre que la pâte de plâtre pur est acide avec un $\mathrm{pH}=6$, le mortier de plâtre est neutre, l'ajout du clinker sulfoalumineux augmente le $\mathrm{pH}$ variant entre 9 et 12 .

Avec le gypse le $\mathrm{pH}$ du mortier s'améliore et varie entre 10 et 12. A noter que le $\mathrm{pH}$ requis pour un béton ordinaire est de l'ordre de 13.

Le temps de prise pour les mortiers de plâtre est très rapide. Par contre le mortier de gypse a un temps de prise supérieur à 3 heures.

Les propriétés mécaniques ont été également examinées. L'évolution de la résistance à la flexion et en compression en fonction de l'âge du mortier (2,7 et 28 jours) a été effectuée pour toutes les formules réalisées.

Les résultats montrent que pour la pâte de plâtre pur et les mortiers de plâtre sans clinker sulfoalumineux, la résistance à la flexion diminue de 2 à 28 jours.

Par contre, pour les mortiers contenant le clinker sulfoalumineux, la résistance à la flexion augmente de 2 à 28 jours. La résistance maximale pour un mortier de plâtre avec clinker sulfoalumineux et un rapport $\mathrm{E} / \mathrm{L}=0.7$ atteint respectivement $2.5 \mathrm{Mpa}$ et $3 \mathrm{Mpa}$.

Concernant la résistance en compression, les résultats montrent que la formule contenant le (plâtre, sable, CSA, $\mathrm{E} / \mathrm{L}=0.7)$ donne les résistances les plus élevées $(\mathrm{RC} 7 \mathrm{j}=10.37 \mathrm{Mpa})$ puis diminue jusqu'à $(\mathrm{RC} 28 \mathrm{j}=8.24$ Mpa). Par contre pour la formule contenant le (plâtre, sable, $\mathrm{CSA}, \mathrm{E} / \mathrm{L}=6$ ) la résistance en compression est croissante de 2 à 28 jours.

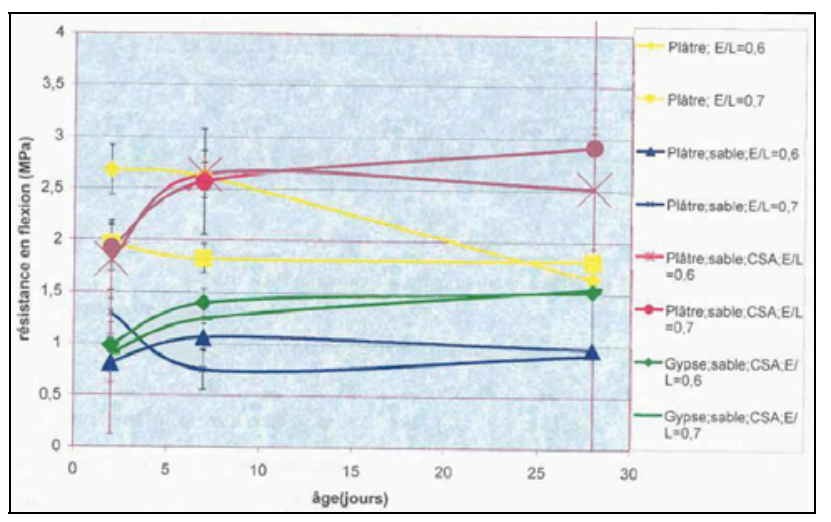

Fig. 1. Resistances en flexion en fonction de l'âge.

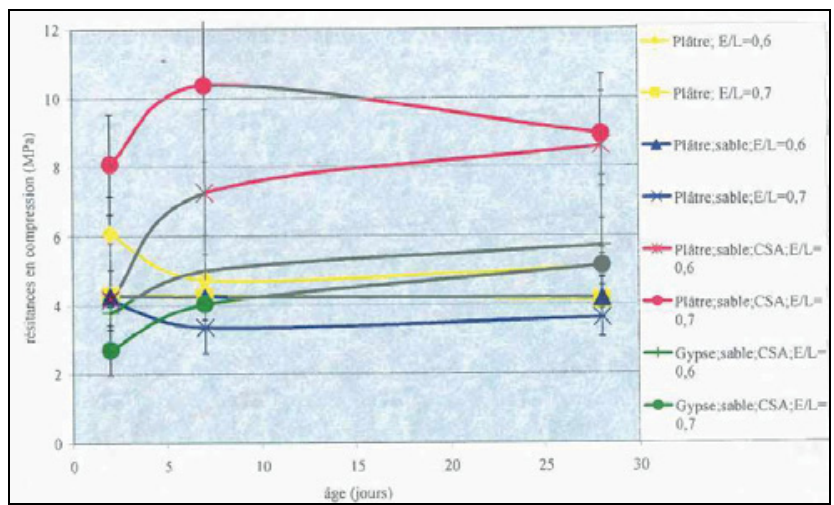

Fig. 2. Resistances à la compression en fonction de l'âge.

A noter que la résistance en compression pour un mortier de plâtre avec clinker sulfoalumineux augmente en augmentant le rapport E/L.

Les propriétés à la tenue à l'eau ont été déterminées en réalisant: l'essai d'absorption capillaire et l'essai de vieillissement accéléré.

Concernant l'essai d'absorption capillaire, les résultats montrent que la variation de masse est moins importante pour les formules contenant du plâtre et du clinker sulfoalumineux ce qui affirme sa faible porosité.

Pour les formules contenant le gypse, l'essai d'absorption capillaire n'a pas beaucoup d'influence sur la résistance 
en compression, or pour les formules contenant le plâtre, la résistance a nettement diminué.

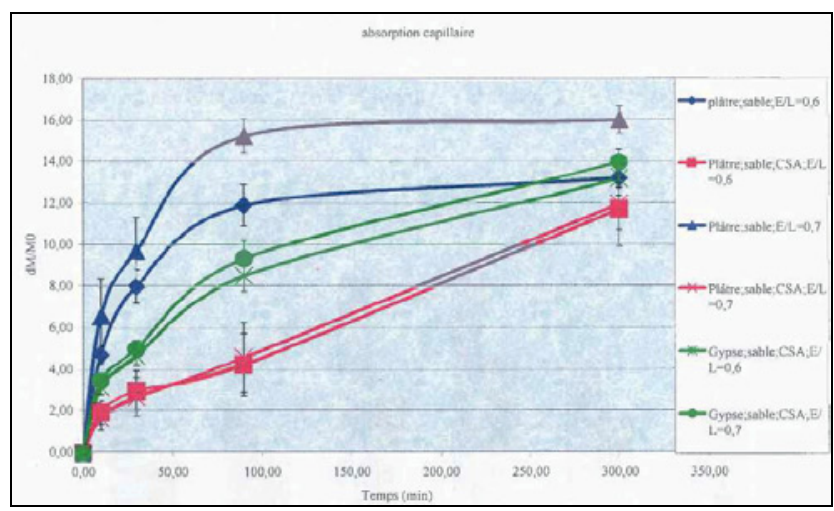

Fig. 3. Variation de la masse pour l'essai d'absorption capillaire

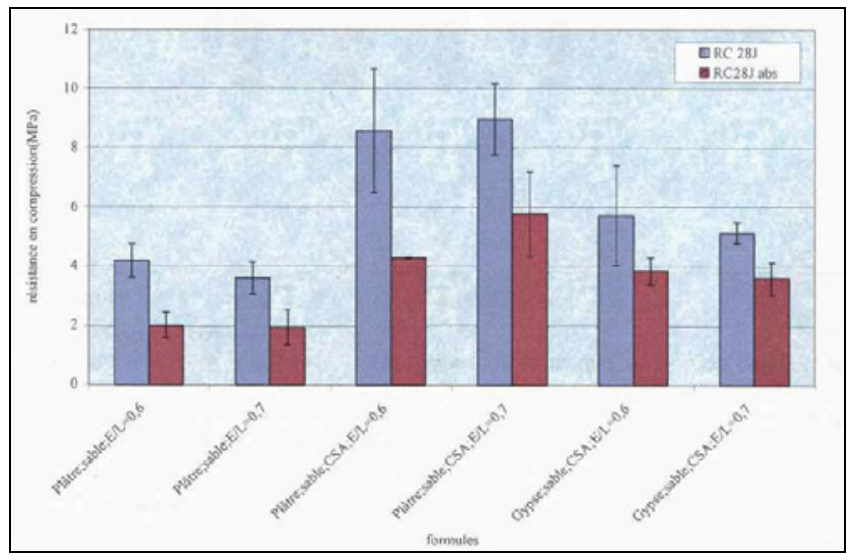

Fig. 4. Comparaison de la résistance en compression des éprouvettes saturées et non-saturées

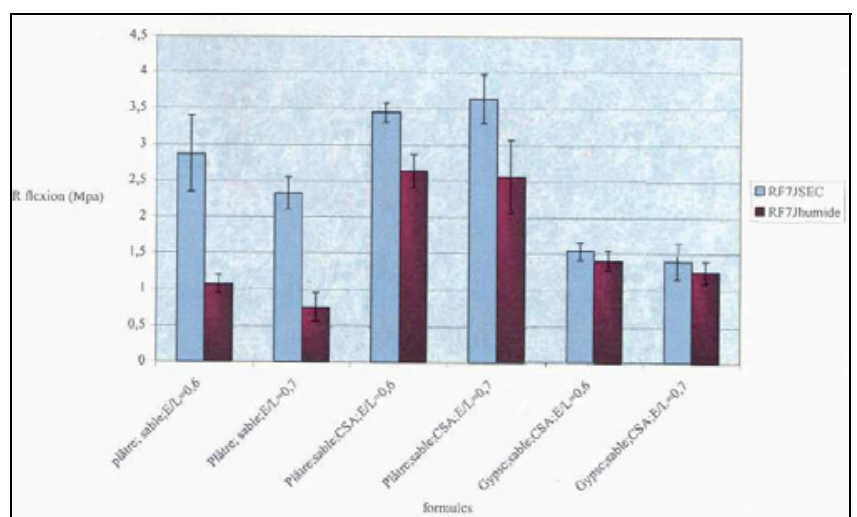

Fig. 5. Resistances en flexion en milieu humide et sec

Les résultats de l'essai de vieillissement accéléré montrent que pour le mortier de plâtre avec clinker sulfoalumineux, la perte de masse se stabilise après la première semaine du cycle de vieillissement accéléré autour d'une valeur de l'ordre de 3\%. Donc, le choix du CSA pour améliorer la tenue à l'eau des mortiers de plâtre est pertinent.

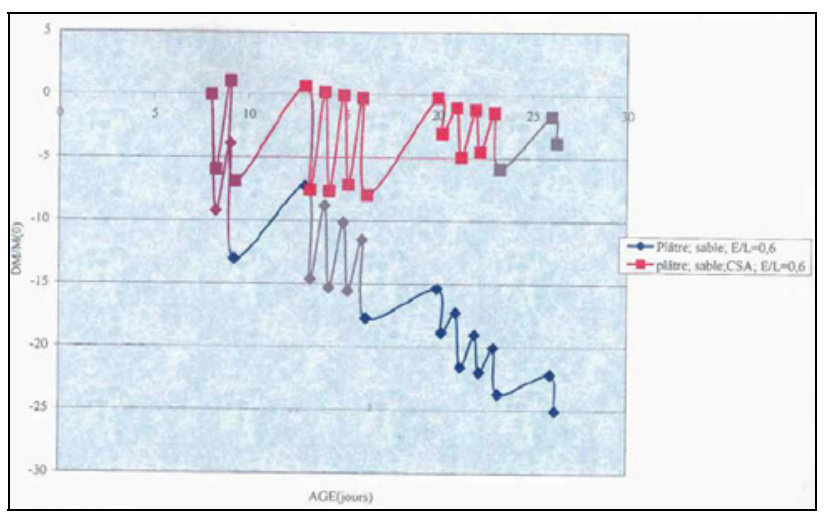

Fig. 6. Variation de la masse pour le $1^{\text {er }}$ cycle de vieillissement

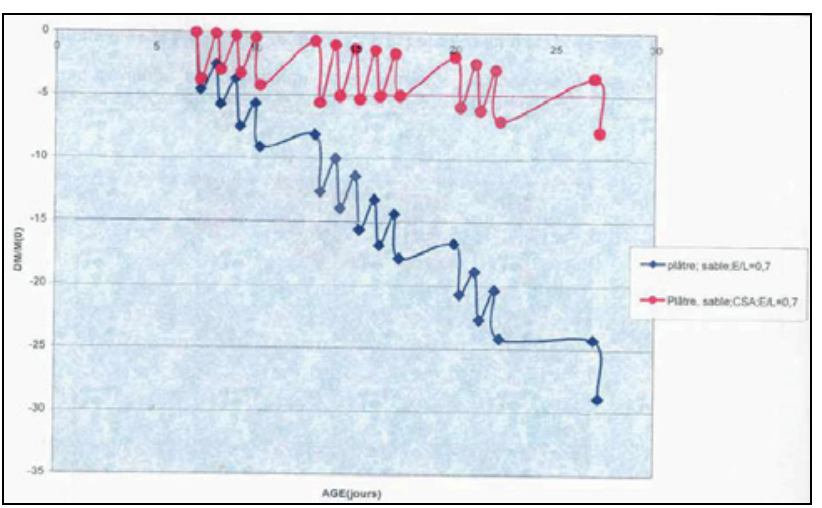

Fig. 7. Variation de la masse pour le $2^{\text {ème }}$ cycle de lessivage

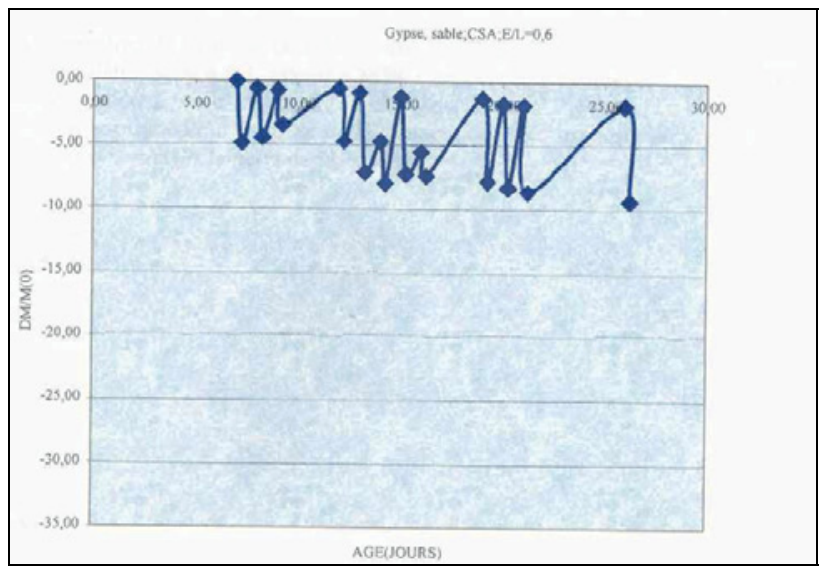

Fig. 8. Variation de la masse pour le $3^{\text {ème }}$ cycle de vieillissement 


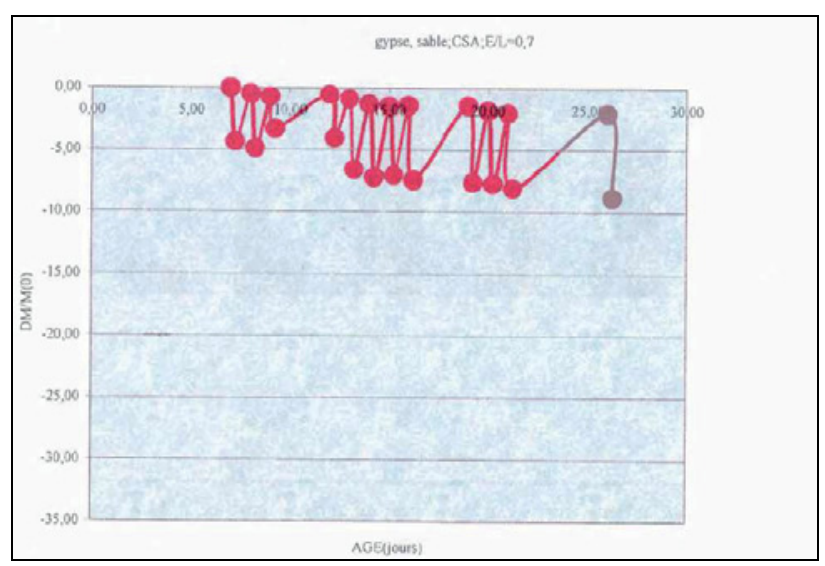

Fig. 9. Variation de la masse pour le 3ème cycle de vieillissement (mortier de gypse avec un rapport $\mathrm{E} / \mathrm{L}=0,6$ )

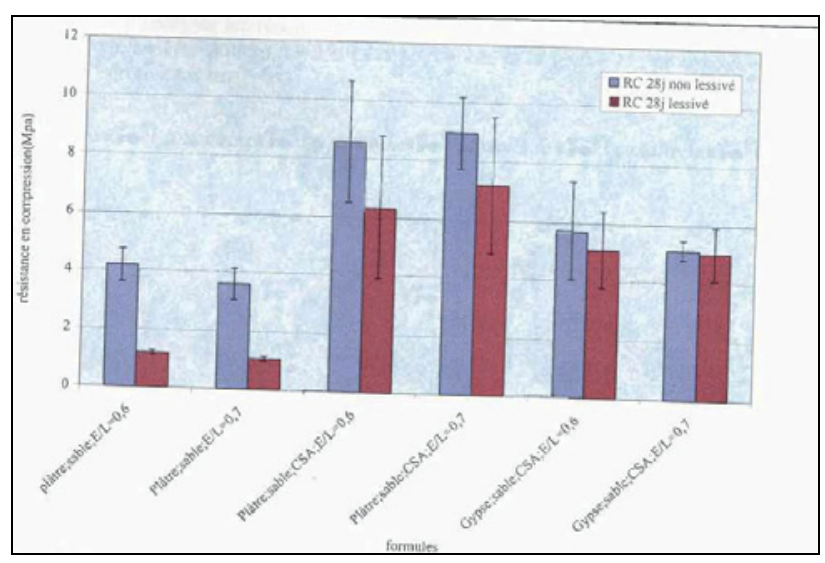

Fig. 10. Comparaison entre les résistances des éprouvettes lessivées et non lessivées

A noter que la variation de résistance en compression d'un mortier de gypse avec clinker sulfoalumineux est faible par rapport au mortier de plâtre avec clinker sulfoalumineux.

L'analyse des résultats obtenus, permet de conclure que la meilleure formule est celle qui contient le plâtre, sable, clinker sulfoalumineux avec un rapport eau sur liant de 0.7. Cette formule représente une bonne tenue à l'eau et de bonne résistance mécaniques au jeune âge. Cependant, elle présente une diminution de la résistance à 28 jours due au processus d'hydratation et un $\mathrm{pH}$ de l'ordre de 9 .

Le mortier avec CSA est un rapport $\mathrm{E} / \mathrm{L}=0.6$ présente un $\mathrm{pH}$ plus intéressant de l'ordre de 12, même si les propriétés mécaniques sont légèrement plus faible.

Afin d'optimiser les résultats obtenues, un logiciel de traitement des données statistiques a été utilisé. Cette analyse montre que les facteurs $\mathrm{E} / \mathrm{L}$ et $\mathrm{CSA} / \mathrm{L}$ ont une grande influence sur les résistances mécaniques, ainsi en augmentant le pourcentage du clinker sulfoalumineux dans le mortier de plâtre avec une quantité d'eau nécessaire à l'hydratation du plâtre, on pourrait obtenir des résistances meilleures. L'optimisation de la formulation du liant à base de plâtre, doit prendre en considération les résistances mécaniques et les résistances à la tenue à l'eau. De tout ce qui précède, la formule optimale est la formule contenant le plâtre, sable, clinker sulfoalumineux avec un rapport eau sur liant de 0.7 .

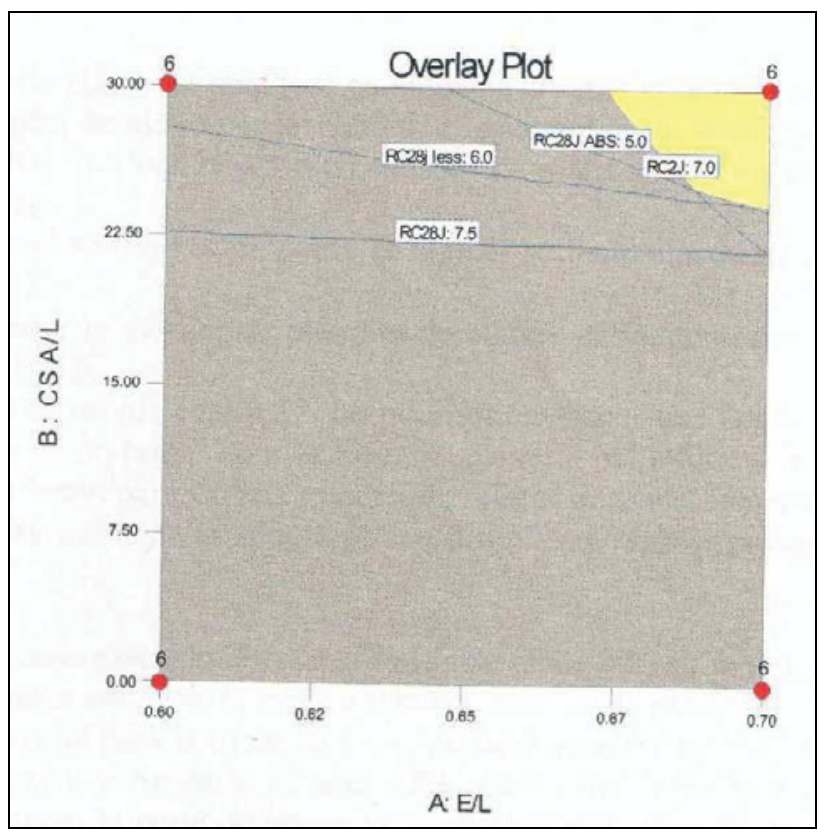

Fig. 11. Domaine d'optimisation des résultats

\section{Conclusion}

Ce travail présente la faisabilité technique d'un liant de plâtre et de clinker sulfoalumineux en examinant l'impact de l'ajout de clinker sulfoalumineux au mortier de plâtre et en étudiant la nécessité de transformer le gypse en plâtre.

Suivant la méthodologie des plans d'expérience, les propriétés rhéologique, mécanique et à la tenue d'eau ont été étudiées. Les résultats de ces essais montrent que l'ajout du sable et du clinker sulfoalumineux améliore le $\mathrm{pH}$ du mortier de plâtre qui atteint 12 pour le mortier de plâtre et de clinker sulfoalumineux avec un rapport eau sur liant de 0.6. Les résultats des essais mécaniques et de tenue à l'eau montrent que l'addition du clinker sulfoalumineux améliore la tenue à l'eau du mortier de plâtre. La perte de masse pour le mortier de plâtre avec clinker sulfoalumineux varie uniquement entre $3 \%$ et $7 \%$, et la perte de résistance en compression est seulement de $20 \%$.

Pour la formule avec $\mathrm{pH}=12$, elle pourrait faire l'objet d'une application renforcé avec des armatures en béton armé. Pour la formulation ayons un $\mathrm{pH}$ inferieur à 12, elle pourrait être renforcé par des fibres (fibres de verre; fibres naturelles).

\section{References}

1. J. Costes-Le plâtre traditionnel et modern (éditions Eyrolles)

2. M. Nolhier- Construire en plâtre

3. M.Ppadakis-Industrie de la chaux et du ciment (éditions Dunod) 
4. F. Bardin-Le plâtre : sa production et son utilisation dans l'habitat (groupe de recherche et d'échange technologiques).

5. D.Daligrand-Le plâtre (Archives techniques de l'ingenieur C910).

6. J.Demonsant- Comprendre et mener des plans d'experiences (AFNOR, 1996).

7. H. Nguyen-Synthèse bibliographique : Clinker sulfoalumineux.

8. A.Dimassi-Synthèse bibliographique: Clinker sulfoalumineux.

9. Older-Special inorganic cement(E \&SPON, New York 200)

10. E.Gartner-Industrially interesting approaches to lowCO2 cements (Cement and Concrete Research 2004).

11. Bétons suivi rhéologique sur mortier de béton equivalent: Projet National Calibé (Avril 2002).

12. Méthodes d'essais des ciments: Détermination des résistances mécaniques Norme Française NF EN 196-1.

13. Technique des essais: détermination du temps de prise sur mortier normal NF P15-431.

14. Methodes d'essai des mortiers pour maçonnerie: determination du coefficient d'absorption d'eau par capillarité du mortier durci NF EN 1015-18.

15. G.Dreux-Nouveau guide du béton (éditions Eyrolles).

16. F.Gorisse-Essais et contrôle des bétons (éditions Eyrolles). 